unit, and the techniques employed were specifically designed to avoid apprehension or discomfort. The catheters used were of fine bore (maximum external diameter 0.6 and $1.8 \mathrm{~mm}$. respectively) and were inserted percutaneously under local anaesthesia. The procedure is comparable to that of establishing an intravenous infusion and no skin incision is used. Flow-guided catheterization of the pulmonary artery at the bedside does not require $\boldsymbol{x}$-ray screening nor is it associated with discomfort. Premedication is therefore not necessary since the patients are not distressed by the procedure.

The patients included in our report were all being monitored haemodynamically and electrocardiographically to provide information of immediate therapeutic value. The pressures recorded through the catheters suggested adverse haemodynamic changes after giving a drug which is in general clinical use as an analgesic for patients with acute myocardial infarction. These changes were not evident on clinical examination. As a result of this investigation the drug was not given to these patients subsequently for the relief of cardiac pain, and we were able to conclude that its general use in the dosage described may possibly be undesirable after acute myocardial infarction. Similar studies performed in this and other units have provided a rational basis for the choice of drug therapy in patients with acute myocardial infarction.

The procedures employed in these studies have been approved by an independent ethical committee of the Royal Postgraduate Medical School. They form an integral part of the programme of work being undertaken in the Medical Research Council's Cardiovascular Unit, with the aim of improving the treatment of patients with acute myocardial infarction.

We would welcome a visit from your correspondents to the Cardiovascular Unit, where they can observe the work we are doing and the results which we have obtained.-We are, etc.,

David Jewitr. BRIAN MAURER.

Department of Medicine, Peter Hubner.

\section{Royal Postgraduate} London $W .12$.

\section{Cost of Treatment}

SIR,-I have received through the post recently an advertisement for Kabinase (pure streptokinase) in which I am told that "anticoagulant therapy is secondary to thrombolytic therapy" in the management of thromboembolism. Indeed, this statement is supported by the many investigative studies that have now been carried out. However, I wonder how many members of the medical profession are aware of the cost of this form of treatment.

Kabinase is supplied in ampoules of 100,000 units, 250,000 units, and 600,000 units and the cost of one ampoule of each is $£ 3$ 14s. 4d., $£ 714$ s. 8d., and $£ 1610$ s. 6d. respectively. Kakkar and his colleagues have described ${ }^{\mathrm{I}}$ a seven-day regimen for treating venous thrombosis with streptokinase. The cost of their regimen, however, is more than $£ 600$ per patient.

I have no wish to restrict the freedom of the pharmaceutical industry with regard to advertising, but I would be happier if every advertisement for a drug or piece of equipment had, by law, to display the cost of the item on that advertisement.-I am, etc.,

\section{Chase Farm Hospital,
Enfield, Middx. \\ REFERENCE \\ Kakkar, V. V., et al., British fournal of Surgery,
1969, 56, 178.}

Alexander P. Ross.

\section{Free Thyroxine Index}

SIR,-We have recently given our experience with Thyopac-3 as an in vitro test of thyroid function. ${ }^{2}$ Like similar methods based on protein-binding of thyroid hormones, Thyopac-3 is affected (as indeed is the protein-bound iodine, P.B.I.) when alterations in protein-binding capacity occur, as in pregnancy or with oestrogen therapy. Such deviations can be misleading, but the true state of affairs may be recognized if both types of test-for example, estimation of the level and protein-binding of thyroid hormone in serum are performed simultaneously. Previously we suggested the use of the "free thyroxine index" (FT,I) in this context. ${ }^{3}$ This by indicating the level of free thyroxine, the determinant of thyroid status, would assist in diagnosis.

We have calculated FT I using the P.B.I. ${ }^{4}$

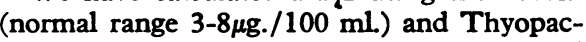
3 (expressed as a ratio to our own control serum; normal range $0.91-1.21$ ) in 237 of the patients previously reported. ${ }^{2}$ There were 108 patients who were shown to be euthyroid, 64 with thyrotoxicosis, and 28 with hypothyroidism and a group of 37 clinically euthyroid women who were either pregnant or taking oestrogen-containing oral contraceptives. Thyroid status was confirmed in the first three groups by thyroid radioiodine uptake and P.B.I., and if necessary by continued observation or by response to specific therapy. The FT, I was obtained by dividing the individual P.B.I. by the respective Thyopac-3 value-for example: $\mathrm{FT}_{4} \mathrm{I}=\frac{\text { P.B.I. }}{\text { Thyopac- }}$

The rationale is as follows: The reaction between thyroid hormone and binding proteins can be expressed: FT + UTBP $\rightleftharpoons$ TBPT where FT, $=$ free thyroxine, UTBP = unsaturated canacity of thyroxine binding proteins, and TBPT $^{4}=$ thyroxine bound to these proteins.

At equilibrium: $\frac{\left(\mathrm{FT}_{1}\right)(\mathrm{UTBP})}{\left(\mathrm{TBPT}_{4}\right)}=\mathrm{K}$, the

dissociation constant, or $\frac{1}{\mathrm{~K}} \mathrm{FT}_{4}=\underset{\mathrm{UTBP}}{\mathrm{TBPT}_{4}}$

P.B.I. can be substituted for TBPT and Thyopac-3 gives an indication of the level of UTBP. That is, $\frac{1}{\mathrm{~K}} \mathrm{FT}_{4}=\frac{\text { P.B.I. }}{\text { Thyopac-3 }}$

The Figure shows the results for P.B.I. and FT $I$ in the groups described. Thyopac3 results have been published previously. ${ }^{2}$ The normal range for FT I $(3.7-8.6)$ was obtained from the euthyroid group. As can be seen $2(3.1 \%)$ of the thyrotoxic and 5 $(17.9 \%)$ of the hypothyroid group had a P.B.I. within the normal range, and 20 $(54.1 \%)$ of the pregnant/oral contraceptive group had an elevated P.B.I. By use of FT I the numbers in the normal range for thyrotoxicosis and hypothyroidism fall to 1 $(1.6 \%)$ and 0 respectively. In addition only $1(2.7 \%)$ in the pregnant/oral contraceptive group was abnormal. Equivalent figures for Thyopac-3 are: thyrotoxic $12.7 \%$ within, hypothyroid $10.8 \%$ within, and pregnant/oral contraceptive $79.1 \%$ outside the normal range. ${ }^{2}$ Not shown on the scatter diagram are the results of FT I for four patients with thyrotoxicosis who were either pregnant (1) or on oral contraceptives (3). These ranged from 11.4 to 15.0 , well outside the normal range.

It is suggested that the "free thyroxine index" is of value when P.B.I. or Thyopac3 alone are misleading.-We are, etc.,

\section{Frederick Clark.}

HAzel J. Brown.

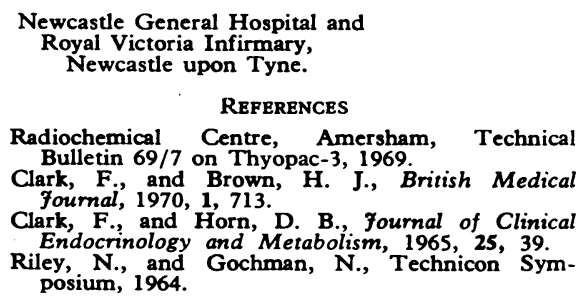

\section{Teething Troubles}

SIR,-In your leading article on teething troubles (11 April, p. 67) you write that $\mathbf{R}$. $S$. Illingworth "stated there is no evidence that teething causes convulsions, fever, bronchitis or diarrhoea." You also state that it is perhaps going too far to say that "teething produces nothing but teeth."

I am always amused at the way we are reluctant to go the whole hog. More and more paediatricians are willing to admit that in their opinion teething causes few symptoms, but they like to cling on to something nebulous and to be able to say an erupting tooth "does seem to cause discomfort."

We seem, even in this enlightened age, to have forgotten that teething is a normal physiological process and therefore is unlikely to produce symptoms. The abnormal signs and symptoms ascribed to teething cover everything that a child can have wrong with him between the ages of six months and two years. Between these ages children are often unwell and the reasons are not difficult to find. Feeding habits change, passive immunity from the mother is waning, and a child is brought into increasing contact with infection. He bites his fingers and on hard objects because he has learnt a new skill and found a nice hole in which to put things. It has nothing to do with teeth.

In fact, babies born with teeth and those who never develop any teeth at all go through exactly the same stages as those who pass through the normal milestones.

I confess quite happily that I am the culprit who, in 1957, wrote "let us admit once and for all, that teething causes only teeth."' I have seen no reason to change my mind. I am still fascinated by the thought of all of us suffering agonies while our teeth erupt like Vesuvius. Surely we ought to resurrect the idea that "growing pains" are real. And for good measure we might add that the growth of nails is painful and the growth of hair quite excruciating. 